\title{
Mating Affects Lifespan Differently in Two Strains of Pseudo-female Caenorhabditis elegans Ruben Lancaster*, Biology
}

\begin{abstract}
Mating is vital for sexually reproducing species, yet the ideal mating strategy for males and females can differ. The ensuing conflict between the sexes - namely, sexual conflict - results in a decrease in population level fitness. The degree of sexual conflict can be affected by the behavior, physiology, and life history of a population. Previous studies in the nematode Caenorhabditis elegans have shown that mating causes lifespan to decrease in pseudo-females and hermaphrodites, which was interpreted as evidence of sexual conflict. However, it is still an open question whether variations in mating condition and strain type can affect the degree of sexual conflict and lifespan decrease. Here, I investigate whether lifespan is affected by mating in conditions other than sexskewed individual mating scenarios used in previous work. I conducted population-based mating assays in two different strains of $C$. elegans using both natural and male-skewed sex ratios. Counter to expectations, I found no effect of mating on lifespan in a wild isolate of $C$. elegans, while virgins from a canonical laboratory strain had a decreased lifespan relative to their counterparts mated in groups. My data offers a counterpoint to the literature, which agrees that mating universally decreases the lifespan of $C$. elegans pseudo-females and hermaphrodites. These results highlight the flexibility of reproductive costs and the importance of life histories in experimental populations.
\end{abstract}

\section{INTRODUCTION}

Males and females of the same species can have different optimal mating strategies. For example, in fruit flies, males produce maximum offspring at high re-mating rates, limited by the number of females with which they can mate (Bateman, 1948; Fowler and Partridge, 1989). Females produce maximum offspring at intermediate mating rates, limited by the number of eggs they can lay (Fowler and Partridge, 1989). In another example, male beetles are known to damage female beetle reproductive tracts as a by-product of selection for traits that increase male fertilization success (Arnqvist and Rowe 2013; Hotzy et al, 2012; Morrow et al, 2003). The with departmental honors. They worked in the Phillips Lab as an undergraduate laboratory technician and undergraduate research assistant. Currently, they live in Los Angeles and are applying to graduate school programs with an emphasis on ecology, evolution, ethics, and public health. They are the proud owner of three frogs. Please direct correspondence to rlanca1996@gmail.com. 
disparity between optimal reproductive phenotype and genotype, as well as the resulting negative effect on fitness, is called sexual conflict. This means that an increase in mating success in one sex may not increase the reproductive output of the other sex.

Every mating carries a risk for both sexes, which can include increased probability of predation, direct physical damage to the female, and excessive depletion of stored energy resources. When females mate beyond the mating frequency that maximizes their lifetime reproductive output, their lifespan may decrease (Arnqvist and Rowe, 2013; Fowler, 1989). Here, I investigate lifespan effects of mating scenarios allowing for different rates of re-mating in strains derived from canonical and wild isolate Caenorhabditis elegans.

\subsection{THE CAENORHABDITIS ELEGANS MODEL ORGANISM}

The nematode $C$. elegans offers natural variation in mating systems that can be taken advantage of when studying sexual conflict. Importantly, post-mating lifespan decrease has been observed in hermaphrodites and pseudo-females in the canonical C. elegans strain N2 (Riddle and Gems, 1996; Shi et al, 2014).

C. elegans is a microscopic nematode usually found in compost and rotting vegetation (Felix et al, 2015). The species has a global distribution with genetically distinct strains isolated in different geographic regions (Sterken et al, 2015). This genetic variation is especially useful for studying the varying responses in sexual conflict due to a population's life history. Nematodes are ideal for conducting studies on aging and lifespan because they are easy to maintain in large populations and have short lifespans of around three to four weeks. This allows for highthroughput lifespan studies.

Different species of Caenorhabditis display different types of mating systems, making them a valuable animal for studies on reproduction (Kiontke et al, 2011). In wild-type C. elegans, which reproduce largely through self-fertilization of the hermaphrodite, males occur at a rate of $0.2 \%$ of the population and arise due to meiotic nondisjunction events (Zarkower, 2006). Other species of Caenorhabditis are obligate male-female reproductive populations with approximately equal sex ratios. Therefore, populations can be dominated by hermaphrodites or have approximately equal numbers of males and females, but natural populations never have male-biased sex ratios.

\subsection{SEXUAL CONFLICT AND LIFESPAN IN C. ELEGANS}

Pseudo-female $C$. elegans have been used in previous studies to disentangle the effects of mating and resource utilization due to self-progeny production and to bypass a known effect of lifespan increase during hermaphrodite-selfing that is not present during outcrossing (Carvalho et al, 2012). Pseudo-female worms are created by a deletion of the fog-2 gene (Clifford et al, 2000; Schedl and Kimble, 1988). Wild type hermaphrodite worms produce sperm during the fourth larval stage, and then irreversibly switch to producing oocytes once they reach adulthood (Zarkower, 2006). However, self-sperm production can be stopped by knocking out the gene fog2 in the spermatogenesis pathway, effectively creating pseudo-female worms. Sperm production 
in fog-2 males is unaffected. Because fog-2 worms reproduce solely through sexual reproduction, this deletion results in a population sex ratio of approximately equal males and females, with every oocyte requiring fertilization from the sperm of a male worm.

Studies of aging and longevity follow two main approaches: observing aging and senescence through a mechanistic lens or through the lens of the life-history of an organism (Caswell-Chen et al, 2005). The mechanisms of lifespan decrease in both female and pseudo-female C. elegans are caused by post-mating pathways associated with pheromones, seminal fluid and sperm, and germline-mediated responses leading to somatic collapse (Gems and Riddle, 1996; Shi et al, 2014). While these mechanistic approaches have provided important insights into the functional biology of aging and sexual conflict, a life-history approach holds the potential to reveal additional variation in sexual conflict phenotypes (Caswell-Chen et al, 2005). Such an approach is equally necessary to complement mechanistic studies, and together they provide a powerful tool for understanding the ecological and mechanistic contexts of aging and sexual conflict.

A life history approach requires biologically relevant mating conditions considering mating both in a group context and at natural mating ratios. Previous work in C. elegans has focused on mating pseudo-females in individual mating assays with strongly male-biased sex ratios to ensure that mating occurs. However, this setup does not reflect natural population ratios of one male for every one pseudo-female. These male-biased mating scenarios have not been observed in natural mating conditions and potentially introduce re-mating rates not seen in natural populations.

Confounding factors such as temperature and starvation-induced mating variant genotypes are also present in previous work, making it difficult to isolate decreased lifespan due to mating as a sexual conflict phenotype. Additionally, these studies used the standard laboratory strain N2, which is known to have several laboratory-derived alleles with a broad range of phenotypic effects, including behavioral changes (Sterken et al, 2015). These confounding factors bring into question whether the "mating-induced demise" response is unique to N2 in individual mating assays, or whether this response can be replicated in wild isolates of $C$. elegans as well.

I conducted a series of individual and group matings in one fog-2 natural isolate and one fog2 lab isolate of $C$. elegans to investigate whether mating-induced lifespan decrease is impacted by mating ratios and population setting.

\section{MATERIALS AND METHODS}

\subsection{ELEGANS STRAINS AND MAINTENANCE}

I used two pseudo-female strains of $C$. elegans: JK574 (fog-2(q71) V), the "canonical strain" derived from N2, referred to as N2fog-2 in this paper, and PX632 (P pie-1::TIR-1::mRuby, $\mathrm{I}: 2851009]$; spe-44(fx110[spe-44::degron]) IV; fog-2(fx111) V), which is derived from the wild isolate JU2526 and is genetically distinct from $\mathrm{N2}$, referred to as wildfog-2. Worms were maintained on $60 \mathrm{~mm}$ petri NGM plates at $20^{\circ} \mathrm{C}$ seeded with $200 \mu \mathrm{L}$ OP5O-1 Escherichia coli (Brenner, 1974). 


\subsection{LIFESPAN ASSAY DESIGN}

Lifespans of nematodes were measured using the C. elegans Automated Lifespan Machine (ALM), an automated system comprising office scanners and imaging software, following Stroustrup et al. (2013).

\subsection{GROUP MATINGS}

Nematode cohorts were created following the Caenorhabditis Intervention and Testing Program protocol (unpublished, C. Sedore pers comm.).

I created three cohorts to be mated at different sex ratios: one of only virgin pseudo-females, one of pseudo-females mated at a natural population sex ratio, and one of pseudo-females mated at a ratio of three males to one pseudo-female (Figure 1). All nematodes began in agesynchronized cohorts, achieved by letting parent day-two adult pseudo-females lay eggs on plates for 2 hours before removing parent pseudo-females. Therefore, all nematodes in these cohorts are aged within 2 hours of each other. At 19-20 hours post egg-lay, nematodes have hatched and are larval, but are not yet reproductively mature adults. At this stage it is possible to differentiate the sexes based on distinctive sex characteristics. At this time, pseudo-females in the pre-adult virgin cohort were isolated from males on separate plates to ensure they never mated. The nematodes in the cohort of male-skewed mating ratios were transferred to plates with fifteen females for every forty-five males, a 3:1 male-skewed sex ratio. The nematodes in the cohort destined to mate in natural population ratios were left on the plates in the same ratios in which they were laid. For both natural and male-skewed ratio cohorts, mating began after nematodes became reproductively mature at 26-28 hours. Mating was allowed for 42 hours, then males were removed (Figure 1).

Next, all pseudo-females were moved to plates with $51 \mathrm{mM}$ 5-fluoro-2'-deoxyuridine (FUdR) to arrest the germline and assure progeny were inviable. Note the virgin cohort did not produce progeny but was also placed on FUdR as a control. Pseudo-females were transferred again to fresh FUdR plates 24 hours later to ensure no viable progeny remained.

On day five of adulthood, pseudo-females were transferred to scanner plates at densities of approximately 60 worms per plate and placed on the ALM. Images of each plate were taken every hour. Data collection on worm lifespans using the ALM lasted for 25 days, after which the images were compiled. Software identified when a worm had died and noted this time (Stroustrup et al, 2013). Lifespans were calculated using the day of the egg lay as day o. The experimental design from egg lay to the ALM was the same for all treatments (Figure 1).

This process was repeated for both N2fog-2 and for wildfog-2 nematodes. 


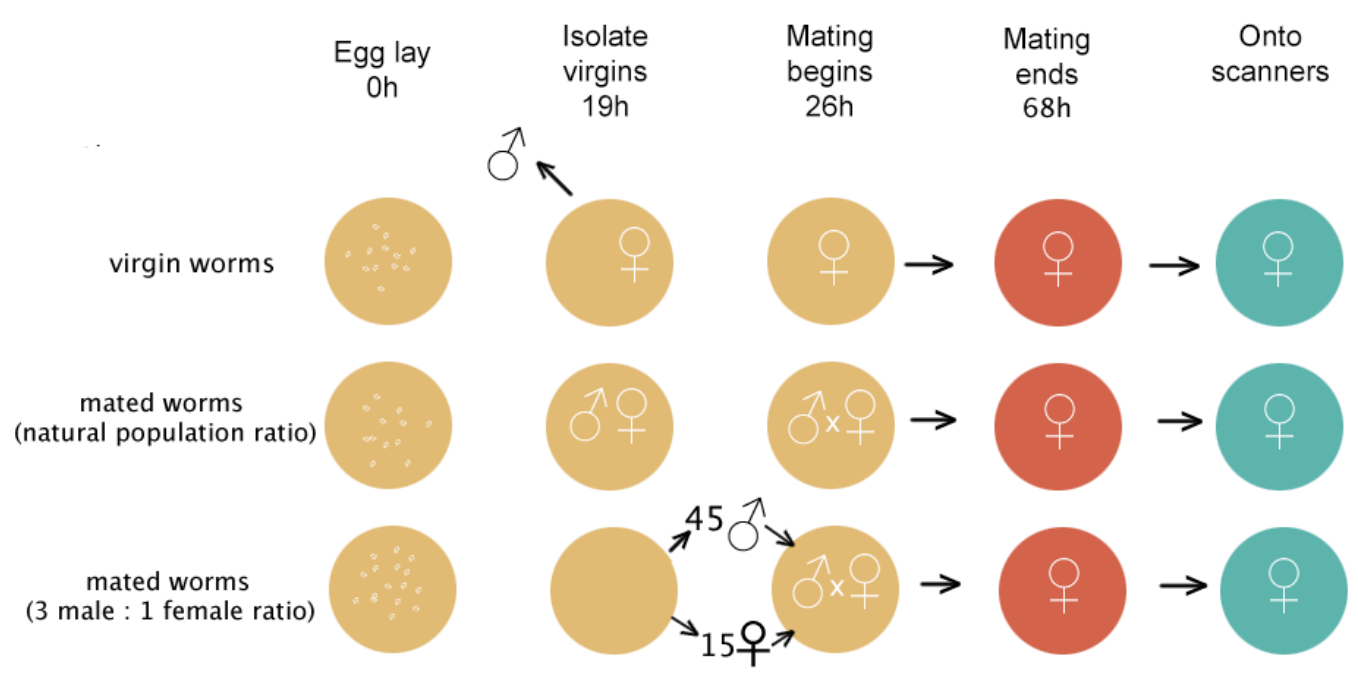

Figure 1: Schematic of experimental design for population mating from egg lay to placement on the C. elegans Lifespan Machine. Circles represent petri plates. Yellow plates, NGM. Red plates, NGM + FUdR. Blue plates, NGM + FUdR on scanners.

\subsection{INDIVIDUAL MATINGS}

Previous work used mating scenarios of one individual female mated to three males in the canonical strain N2, which also had a fog-2 mutation (Shi et al, 2014). I mated nematodes on individual plates of one female and three males to verify a lifespan decrease in my experimental population of N2fog-2 (Figure 2).

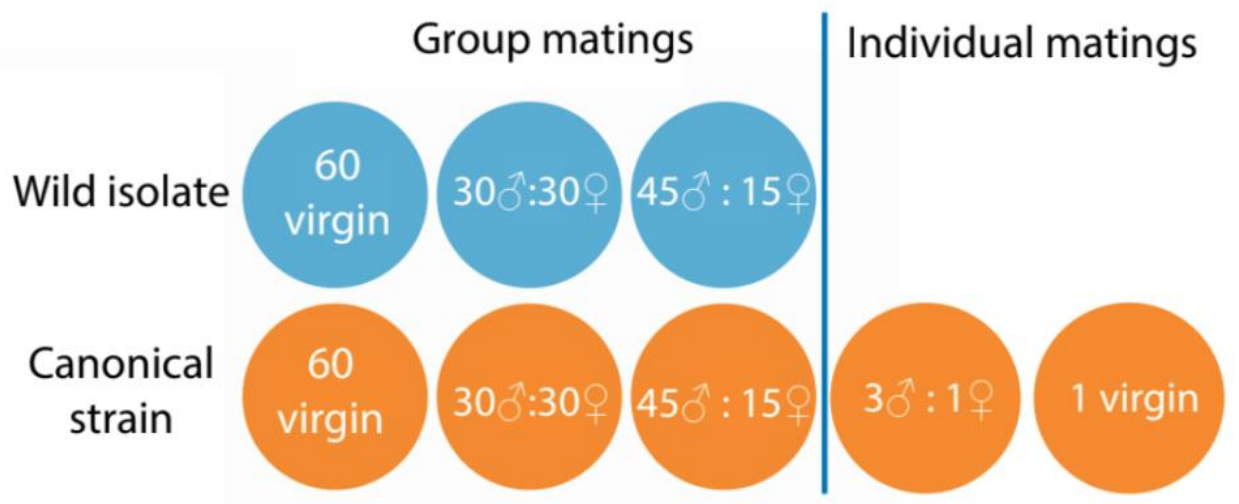

Figure 2: Group and individual matings in different ratios of wild isolate (blue) and canonical strain (orange) populations. Treatments of each mating ratio, 1 female to 1 male or 1 female to 3 males, and virgin worms are performed in the canonical strain and wild isolate.

\subsection{STATISTICAL ANALYSIS}

Lifespan data were analyzed using a linear mixed model in lme4 (Bates et al, 2015) and a mixed effects Cox Proportional Hazards model in coxme (Therneau, 2018) in the R Statistical Package (R Core Team, 2013). 


\section{RESULTS}

In N2fog-2, pseudo-females mated in groups of male-skewed ratios had significantly longer lifespans than virgin pseudo-females mated in both group $(p<0.001)$ and individual $(p<0.05)$ assays (Figure $3 \mathrm{~A}$ ). The shape of the lifespan curves of N2fog-2 virgins is intriguing as they do not mimic the lifespan curve of mated worms, due to an abnormal linear decrease in cohort survivorship by day 20 (Figure 3A).

There were no significant differences between mated and virgin worms in the wildfog-2 strain, even at skewed mating ratios $(p<0.001)$ (Figure 3B).

Between strains, lifespans of N2fog-2 virgins were significantly shorter than wildfog-2 mated at a one-to-one sex ratio $(p<0.001)$ (Figure $3 \mathrm{C}$ ). Virgin worms of each strain had significantly different lifespans $(p<0.01)$. The shape of lifespan curves of N2fog-2 and wildfog-2 strains appeared different, but the power of this study was insufficient to define this difference.

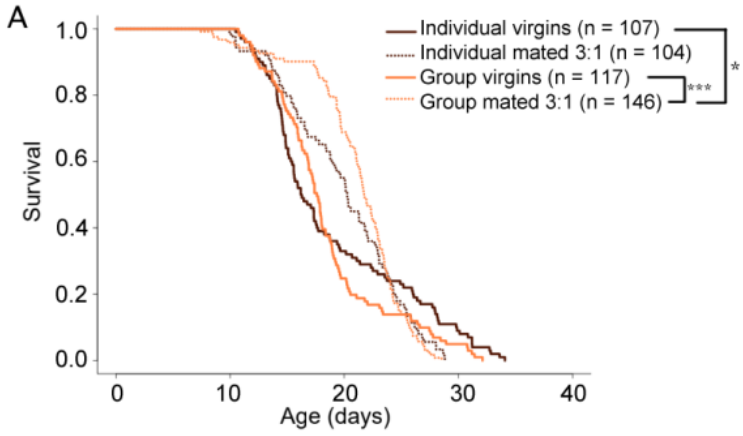

B

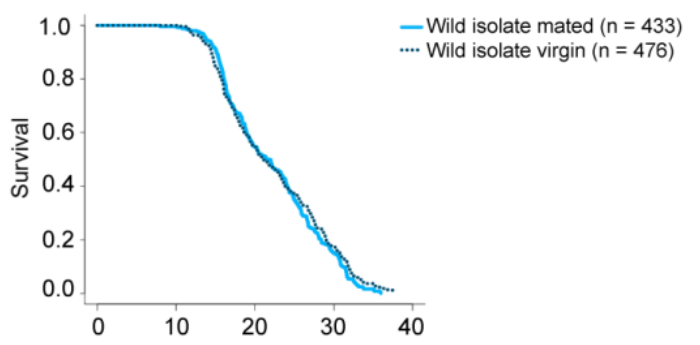

C

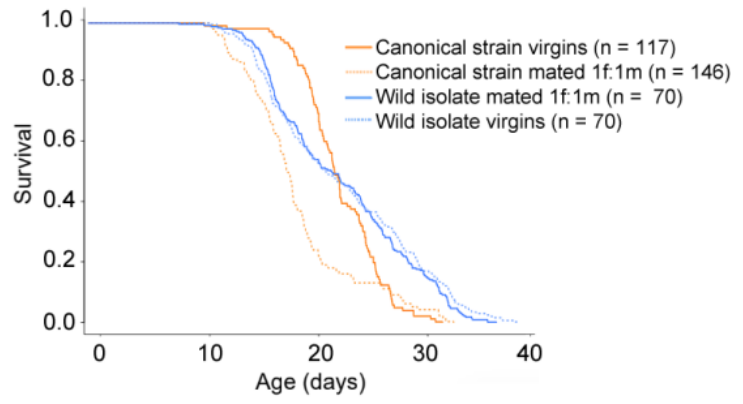

Figure 3: Lifespans of the treatments. A. N2fog-2 analysis. Mated (3:1) and virgin worm lifespans are significantly different in both group $(p<0.001)$ and individual $(p<0.05)$ analyses. Whether the worm was in the individual or group 


\begin{abstract}
cohort had no significant effect on lifespan. B. Wildfog-2 analyses. Mated (1:1) and virgin worms do not differ significantly in lifespan. Data from three pooled replicates. C. Lifespans of N2fog-2 ("canonical strain") and wildfog-2 ("wild isolate") worms in group mating treatments.
\end{abstract}

To investigate whether the fog-2 mutation itself led to changes in lifespan, I compared lifespans of hermaphroditic ancestors. Wildfog-2 lives are shorter than their hermaphrodite ancestor $(p<0.05)$ (C. Sedore pers. comm.). The N2 ancestral hermaphrodite has a median lifespan range of 15 to 17 days (Riddle and Gems, 2000), compared to 17 days (virgin) and 22 days (mated) of the N2fog-2 strain.

\title{
4. Discussion
}

I compared lifespans of mated and virgin worms in two different strains of $C$. elegans using an Automated Lifespan Machine (Stroustrup et al, 2013). I found no significant differences between mated and virgin worms in a wild isolate-derived strain, even at male-skewed mating ratios. In a canonically derived strain, however, virgin worms had significantly shorter lifespans than worms mated at a ratio of three males to one female, contrary to previous work.

The responses to mating stress differed in each strain. This suggests the degree of lifespan decrease is strain-dependent and highlights the importance of using strains outside of the N2 reference strain. The N2 strain is known to have many laboratory adaptations (Sterken et al, 2015), and it is possible that the response of N2 is not reflective of responses of C. elegans strains drawn directly from nature. Previous studies in N2 have shown decreases in lifespan after mating in both hermaphrodite and pseudo-female C. elegans, while this study showed the opposite effect of increased lifespan relative to virgin pseudo-females.

One interpretation of this effect is simply that life history may dictate responses to mating stress. The previous study that investigated fog-2 lifespans used a different point mutation to create the null phenotype (Shi et al, 2014). This could demonstrate pleiotropy of a single mutation, such that each point mutation has multiple unique phenotypic effects, resulting in phenotypic changes beyond a spermless phenotype. These differences in lifespan between strains are in agreement with the differences observed between not only various wild isolate strains and N2 strains, but notably within N2 strains from a common ancestor themselves when maintained in different laboratories (Riddle and Gems, 2000).

Another plausible explanation of this difference is the main deviation in methodology, as the use of the ALM could have generated a slightly different response than that previously observed. There are numerous differences between the ALM and manual assays, but two possibilities stand out. First, it is possible previous studies found mated worms have decreased lifespans due to the combined stresses of mating and daily picking in manual assays that is not present when using the ALM. This would explain a lack of decrease in mated worm lifespans. Second, there may be population effects of pheromone signaling present on the ALM that are not present when worms are isolated on plates. C. elegans uses pheromone signaling for communicating about food availability, population density, and sexual reproduction; this signaling is known to induce physiological, behavioral, and lifespan changes (Shi et al, 2017). The effects of an increased 
concentration in pheromone signaling may alter lifespan as mated worms and virgin worms respond to pheromones differently (Borne et al, 2017). A study investigating the effects of mating when living as a population on the ALM, which has an enclosed and dynamic environment of changing oxygen levels and bacteria availability, compared to the relatively static environment of transferring worms to fresh plates, would give insight into this difference.

\subsection{AGING AND THE “FEMALE-LIKE” STATE}

Previous studies have shown that changes in gene expression associated with the spermless hermaphrodite, termed the "female-like" state, are also associated with aging and physiological changes (Angeles-Albores et al, 2017). It is possible that a spermless state for pseudo-females induces a fast-aging phenotype unique to the "female-like" state observed in this study. However, while the female-like state may explain fog-2 associations with aging phenotypes, this explanation fails to reconcile the difference between fast and slow aging in this and previous studies (Shi et al, 2014).

Previous experimental evolution using the same strain of N2fog-2 (JK574) to generate highconflict male-female mating systems showed a trend of increased sexual conflict over evolutionary time (Palopoli et al, 2015). N2fog-2 worms may not be evolutionarily adapted to both mating in a male-female population and the self-spermless state induced by a fog-2 mutation. This would create a difference between mated and virgin treatments.

I surveyed only a single point in evolutionary time, giving a static window into the sexual conflict phenotypes present in these two populations of fog-2 worms. It is likely that as evolutionary time progresses from onset of the fog-2 mutation, C. elegans will show differential responses to this artificially imposed mating system. Sexual conflict phenotypes depend largely on the type of mating system, so the change from self-fertilization to outcrossing carries with it the potential for new and distinct sexual conflict phenotypes to emerge.

\section{CONCLUSIONS}

To further investigate the potential for evolutionary adaptation, I would follow an experimental evolution paradigm with N2fog-2 pseudo-females evolved in a sperm-limited state and compare lifespan responses to mating after several generations.

Explorations in a wider variety of strains of $C$. elegans will shed light on the potentially varied effects of life history on post-mating longevity. Understanding the type of traits under sexual conflict and the ensuing fitness decrease across strains and mating systems will provide important information on mating dynamics in this genus. An increased understanding of strain-dependent mating effects may provide an answer to irreconcilable differences in results previously described in the literature (Riddle and Gems, 1996; Voorhies, 1992). Studies like this and the ones proposed are important for our understanding of how intertwined sexual conflict, mating behavior, and longevity are in Caenorhabditis nematodes.

Unique life histories can cause differential sexual conflict phenotypes. The variation present 
among sexual conflict phenotypes even within the same species and mating system cannot be taken for granted. Broadly speaking, the potential for variation must be taken into account when investigating sexual conflict phenotypes both in the lab and in the field, and caution must be taken when extrapolating sexual conflict phenotypes to a species as a whole.

\section{ACKNOWLEDGEMENTS}

I would like to thank Dr. Patrick Phillips and Katja Kasimatis for their guidance, insight, and support over the course of this project; Christine Sedore for her help and instruction; Caenorhabditis Intervention and Testing Program technicians for help with experimental procedures; and everyone in the Phillips Lab for the great opportunities and learning experiences I've had in my time there. This work was funded by the NIH (Grant Number Ro1 GM102511 to PCP) and an UnderGrEBES Research Award.

\section{REFERENCES}

Angeles-Albores D, Leighton DHW, Tsou T, Khaw TH, Antoshechkin I, Sternberg PW. 2017. The C. elegans female state: Decoupling the transcriptomic effects of aging and sperm-status. G3 (Bethesda) 7(9): 2969-2977.

Arnqvist G and Rowe L. 2013. Sexual conflict. Princeton University Press.

Bates D, Machler M, Bolker B, Walker S. 2015. Fitting Linear Mixed-Effects Models Using lme4. Journal of Statistical Software 67(1):1-48.

Bateman, A. J. 1948. Intra-sexual selection in Drosophila. Heredity 2:349-368.

Borne F, Kasimatis K, Phillips P. 2017. Quantifying male and female pheromone-based mate choice in Caenorhabditis nematodes using a novel microfluidic technique. PLoS ONE 12(12): e0189679.

Brenner S. 1974. The genetics of Caenorhabditis elegans. Genetics 77:71-94.

Carvalho S, Phillips P, Teotonio H. 2014. Hermaphrodite life history and the maintenance of partial selfing in experimental populations of Caenorhabditis elegans. BMC Evolutionary Biology 14:117.

Caswell-Chen EP, Chen J, Lewis EE, Douhan GW, Nadler SA, Carey JR. 2005. Revising the Standard Wisdom of C. elegans Natural History: Ecology of Longevity. Sci. Aging Knowl. Environ. (40) pe30(2005).

Clifford R, Lee MH, Nayak S, Ohmachi M, Giorgini F, Schedl T. 2000. FOG-2, a novel F-box containing protein, associates with the GLD-1 RNA binding protein and directs male sex determination in the C. elegans hermaphrodite germline. Development 127: 5265-5276.

Cook DE, Zdraljevic S, Tanny RE, Seo B, Riccardi DD, Noble LM, Rockman MV, Alkema MJ, Braendle C, Kammenga JE, Wang J, Kruglyak L, Félix MA, Lee J, Andersen EC. 2016. The 
Genetic Basis of Natural Variation in Caenorhabditis elegans Telomere Length. Genetics 204(1):371-383.

Felix MA and Frezal L. 2015. The Natural History of Model Organisms: C. elegans Outside the Petri Dish. eLIFE 4:e05849.

Fowler K and L Partridge. 1989. A cost of mating in female fruitflies. Nature 338:760-761.

Hotzy C, Polak M, Rönn JL, Arnqvist G. 2012. Phenotypic engineering unveils the function of genital morphology. Curr. Biol. 22: 2258-2261.

Kasimatis K, Moerdyk-Schauwecker M, Phillips P. 2018. Auxin-Mediated Sterility Induction System for Longevity and Mating Studies in Caenorhabditis elegans. G3: Genes, Genomes, Genetics 8:2655-2662.

Kiontke KC, Félix MA, Ailion M, Rockman MV, Braendle C, Pénigault JB, Fitch DH. 2011. A phylogeny and molecular barcodes for Caenorhabditis, with numerous new species from rotting fruits. BMC Evolutionary Biology 11:339.

Morrow EH, Arnqvist G, Pitnick S. 2003. Adaptation versus pleiotropy: why do males harm their mates? Behav. Ecol. 14:802-806.

Palopoli MF, Peden C, Woo C, Akiha K, Ary M, Cruze L, Anderson JL, Phillips PC. 2015. Natural and experimental evolution of sexual conflict within Caenorhabditis nematodes. BMC Evolutionary Biology 15:93.

R Core Team. 2013. R: A language and environment for statistical computing. R Foundation for Statistical Computing, Vienna, Austria. URL http://www.R-project.org/.

Riddle DL and Gems D. 2000. Defining wild-type life span in Caenorhabditis elegans. J Gerontol A Biol Sci Med Sci. 55(5):B215-9.

Riddle DL and Gems D. 1996. Longevity in Caenorhabditis elegans reduced by mating but not gamete production. Nature 379(6567):723-5.

Schedl T and Kimble J. 1988. fog-2, a germ-line-specific sex determination gene required for hermaphrodite spermatogenesis in Caenorhabditis elegans. GENETICS 119(1): 43-61.

Shi C and Murphy CT. 2014. Mating Induces Shrinking and Death in Caenorhabditis Mothers. Science 343(6170):536-40.

Shi C, Runnels AM, Murphy CT. 2017. Mating and male pheromone kill Caenorhabditis males through distinct mechanisms. eLIFE 6:e23493.

Sterken MG, Snoek LB, Kammenga JE, Andersen EC. 2015. The laboratory domestication of $C$. elegans. 2015. Trends Genet. 31(5):224-31. 
Stroustrup N, Ulmschneider B, Nash Z, Lopez-Moyado I, Apfeld J, Fontana W. 2013. The Caenorhabditis elegans Lifespan Machine. 2013. Nat. Methods 10: 665-670.

Therneau T. 2018. Coxme: Mixed Effects Cox Models. URL http://CRAN.Rproject.org/package $=$ coxme

Ting J, Woodruff G, Leung G, Shin N, Cutter A, Haag E. 2014. Intense Sperm-Mediated Sexual Conflict Promotes Reproductive Isolation in Caenorhabditis Nematodes. PLoS Biol 12(7): e1001915.

Voorhies W. 1992. Production of sperm reduces nematode lifespan. Nature 360:456-458.

Zarkower D. 2006. Somatic sex determination. WormBook, ed. The C. elegans Research Community, WormBook. 